\title{
Network Grammars, Communication Behaviors and Automatic Verification
}

\author{
Ze'ev Shtadler Orna Grumberg \\ Computer Science Department \\ The Technion \\ 32000 Haifa, Israel. \\ steedetechunix.bitnet orna@techsel.bitnet
}

\section{Introduction}

A typical distributed algorithm for communicating processes is designed to be applicable to a family of networks with similar topology. Such a topology has some finite number of process types and different number of processes. A few examples are: choosing a leader in a ring (of any size) [L], mutual exclusion (in a ring) [D], distributed termination detection (in any connected network [F], or in a ring [FRS]). An algorithm of this kind can be described by associating with each process type a program with a specified set of communication ports, together with rules to combine processes of these types (by pairing ports) to admissible networks.

In many cases, the program of each of the processes is finite-state. Thus, application of such an algorithm to a specific network is also a finite-state program. Hence, an algorithm of this kind can be viewed as an infinite family of finite-state programs.

Automatic verification of finite-state distributed programs by means of temporal logic model checking has been widely investigated $[\mathrm{CES}],[\mathrm{LP}],[\mathrm{QS}],[\mathrm{EL}],[\mathrm{B}],[\mathrm{SC}]$ and successfully used [BCDM], [RRSV], [CBBG], [DC], [MC]. A model checking algorithm determines whether a finite-state distributed program satisfies a temporal logic formula by searching all possible paths in the global state graph induced by the program.

Recently, the problem of automatic verification of infinite families of finite-state programs was studied by several researchers ([CG], [SG]). However, these works dealt only with algorithms designed for a very restricted class of network topologies. More importantly, they didn't take advantage of the fact that every such infinite family of programs originates from one distributed algorithm. We exploit this observation to suggest a formalism for the description of algorithms that is, on the one hand, close to the designer's view of a distributed algorithm and, on the other hand, facilitates automatic reasoning about the described algorithm. We believe that our formalism will help to significantly narrow the gap between algorithm design and algorithm verification. 
The main contributions of this paper are:

- We suggest a new formalism for the description of algorithms designed for networks with similar topology and different numbers of processes. Our formalism handles network topologies consisting of a fixed number of process types, each of which has a fixed number of communication ports.

- Based on this formalism, we develop a new method for the automatic verification of algorithms described by means of that formalism.

The formalism that we suggest is a context-free network grammar that derives networks of processes. With each derived network, a finite-state program is associated. In a network-grammar, the nonterminals represent subnetwork types (with possibly unpaired ports). The initial variable $T$ represents the network type (with no unpaired ports), and terminals represent (instances of) process types.

Following is an example of a grammar that generates a family of ring networks with any number of processes. For simplicity, the processes are all of the same type $a$. Both the nonterminal $A$ and the terminal $a$ have ports $L$ and $R$, representing the left port and the right port, respectively. The right hand side of the production rules specify how ports are paired.

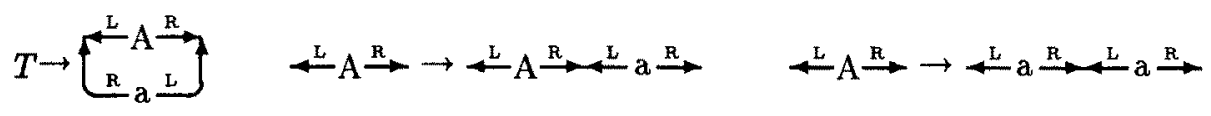

The derivation of a ring of size 4 in the grammar above is:

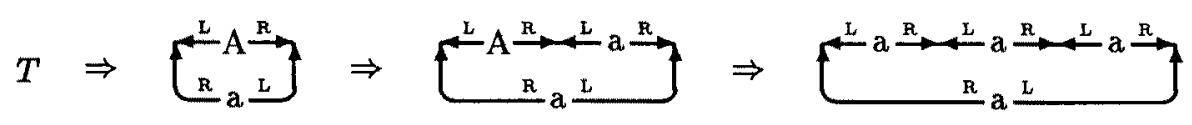

Another important notion presented here is the communication behavior of a subnetwork $P$, given its environment $Q$ (which is also a subnetwork). The communication behavior of $P$ given $Q$, denoted by $P: Q$, is a new subnetwork, which records all the communications of $P$, which are in accord with $Q$, and represents all the others by some dummy communications.

The communication behavior is used as the main tool for reasoning about algorithms defined by means of network grammars. Its definition is motivated by the following observation: when replacing in a network $N=P \| Q$ the subnetwork $P$ with another subnetwork $P^{\prime}, P \| Q$ and $P^{\prime} \| Q$ an equivalent (to be formally defined later) if $P$ and $P^{\prime}$ have the same communication behavior given the same environment $Q$.

Following [CG] we would like to show that all programs derived from a given algorithm, satisfy the same specification, written in a specification language presented below. Showing that, we will be able to prove that an algorithm satisfies some specification in general by considering only one of its small derived programs. For any given algorithm, we employ induction on the derivation of its networks by a network-grammar to prove that all the derived programs are equivalent. 
As the specification language we introduce $\mathrm{LTL}^{2}$, a two-leveled linear-time temporal logic. Its basic formulas are any Indexed Linear Temporal Logic (ILTL) formulas, similar to those defined in [CGB], except that linear-time operators are used instead of branching-time operators. The logic $\mathrm{LTL}^{2}$ is defined by applying a second level of lineartime operators. A typical LTL ${ }^{2}$ formula is:

$$
\mathbf{A F}\left(\bigvee_{i} \mathbf{A G F} a_{i} \rightarrow \bigwedge_{j} \mathbf{A F} b_{j}\right)
$$

where $\bigvee_{i} \mathbf{A G F} a_{i}$ and $\bigwedge_{j} \mathbf{A F} b_{j}$ are its basic ILTL components. This formula is satisfied by a program provided that along every computation of the program eventually the following will be true: if for some process $i, a_{i}$ holds infinitely often along every computation, then for every process $j$, along every computation, $b_{j}$ will eventually hold. A model for our logic is a labeled state transition graph or Kripke structure that represents the possible global state transitions of some network of finite-state processes.

Our paper is organized as follows: Section 2 describes processes, networks and network grammars. Section 3 presents the logic LTL $^{2}$ and its semantics with respect to Kripke structures. Two notions of equivalence are defined over Kripke structures, and are shown to imply each other. Section 4 introduces the communication behavior of a process given its environment, and shows how communication behaviors are used for algorithms verification. Section 5 presents a procedure to determine if an algorithm given by a network grammar satisfies an $\mathrm{LTL}^{2}$ specification.

\section{Processes and Networks}

\subsection{Combining Processes}

Our model of communication is described by means of synchronization communications along channels. The model is similar both to CCS combination [M] and to OCCAM's model of communications [I]. Each process is associated with a set of communication action names (referred to also as ports). $\epsilon$ denotes the non-communication (internal) actions of a process. When two processes $P$ and $Q$ are combined to construct a new process $P \| Q$, some of their ports are paired together. A pair of ports represents a channel of communication between $P$ and $Q$. The channel is used merely for synchronization, i.e. no data is transferred. The unpaired ports of $P$ and $Q$ become the ports of $P \| Q$. A process with no unpaired ports is called a network.

A process $P$ is a 7 -tuple, $P=\left\langle A C T_{P}, A P_{P}, I_{P}, S_{P}, R_{P}, s_{0} P, L_{P}\right\rangle$, where

- $A C T_{P}$ is a finite set of actions (ports) that does not contain $\epsilon$.

- $A P_{P}$ is a finite set of atomic propositions.

- $I_{P}$ is a finite set of indices.

- $S_{P}$ is a finite set of states.

- $R_{P} \subseteq S_{P} \times\left(A C T_{P} \cup\{\epsilon\}\right) \times S_{P}$ is a labeled transition relation. We write $s_{1} \stackrel{a}{\rightarrow} s_{2}$ to indicate that $\left(s_{1}, a, s_{2}\right) \in R_{P}$. 
- $s_{0} P$ is the initial state.

- $L_{P}: S_{P} \rightarrow 2^{\left(A P_{P} \times I_{P}\right)}$ is the function that labels each state with a set of indexed atomic propositions. We will write $a_{i}$ instead of $(a, i)$.

Let $P$ and $Q$ be two processes, with disjoint sets of indices, $I_{P}$ and $I_{Q}$. Let $N_{P}$ : $A C T_{P} \rightarrow A C T$ and $N_{Q}: A C T_{Q} \rightarrow A C T$ be two 1-1 renaming functions, for some set of actions $A C T$. We say that two ports, $a_{P}$ and $a_{Q}$, are paired together if $N_{P}\left(a_{P}\right)=$ $N_{Q}\left(a_{Q}\right)$. The combination of $P$ and $Q$ using $N_{P}$ and $N_{Q}$, is a new process, $P \| Q=$ $\left\langle A C T_{P \| Q}, A P_{P \| Q}, I_{P \| Q}, S_{P \| Q}, R_{P \| Q}, s_{0} P \| Q, L_{P \| Q}\right\rangle$, where

$$
\begin{aligned}
& A C T_{P \| Q}=\left(N_{P}\left(A C T_{P}\right) \cup N_{Q}\left(A C T_{Q}\right)\right)-\left(N_{P}\left(A C T_{P}\right) \cap N_{Q}\left(A C T_{Q}\right)\right) \\
& A P_{P \| Q}=A P_{P} \cup A P_{Q} \\
& I_{P \| Q}=I_{P} \cup I_{Q} \\
& S_{P \| Q}=\left(S_{P} \times S_{Q}\right) \\
& L_{P \| Q}\left(\left(s_{P}, s_{Q}\right)\right)=L_{P}\left(s_{P}\right) \cup L_{Q}\left(s_{Q}\right) \\
& s_{0 P \| Q}=\left(s_{0 P}, s_{0 Q}\right)
\end{aligned}
$$

$$
\begin{aligned}
& R_{P \| Q}= \\
& \begin{array}{l|l}
\left\{\left(s_{P}, s_{Q}\right), \epsilon,\left(s_{P}^{\prime}, s_{Q}^{\prime}\right)\right) & \exists a_{P}, a_{Q}:\left(s_{P}, a_{P}, s_{P}^{\prime}\right) \in R_{P},\left(s_{Q}, a_{Q}, s_{Q}^{\prime}\right) \in R_{Q} \\
& \text { and } \left.N_{P}\left(a_{P}\right)=N_{Q}\left(a_{Q}\right)\right\} \cup \\
\left\{\left(\left(s_{P}, s_{Q}\right), \epsilon,\left(s_{P}^{\prime}, s_{Q}\right)\right)\right. & \left.\left.\mid s_{P}, \epsilon, s_{P}^{\prime}\right) \in R_{P}\right\} \cup \\
\left\{\left(\left(s_{P}, s_{Q}\right), \epsilon,\left(s_{P}, s_{Q}^{\prime}\right)\right)\right. & \left.\left(s_{Q}, \epsilon, s_{Q}^{\prime}\right) \in R_{Q}\right\} \cup \\
\left\{\left(\left(s_{P}, s_{Q}\right), N_{P}\left(a_{P}\right),\left(s_{P}^{\prime}, s_{Q}\right)\right)\right. & \left.\mid N_{P}\left(a_{P}\right) \notin N_{Q}\left(A C T_{Q}\right) \text { and }\left(s_{P}, a_{P}, s_{P}^{\prime}\right) \in R_{P}\right\} \cup \\
\left\{\left(\left(s_{P}, s_{Q}\right), N_{Q}\left(a_{Q}\right),\left(s_{P}, s_{Q}^{\prime}\right)\right)\right. & \left.\mid N_{Q}\left(a_{Q}\right) \notin N_{P}\left(A C T_{P}\right) \text { and }\left(s_{Q}, a_{Q}, s_{Q}^{\prime}\right) \in R_{Q}\right\}
\end{array}
\end{aligned}
$$

Sometimes it will be useful to use a renaming function that leaves the names of the ports unchanged. We then use the identity renaming function, denoted by $\mathcal{I}$.

The empty process $\emptyset=\left\langle A C T_{\emptyset}, A P_{\emptyset}, I_{\emptyset}, S_{\emptyset}, R_{\emptyset}, s_{0}, L_{\emptyset}\right\rangle$, is the process for which $A C T_{\emptyset}=$ $A P_{\emptyset}=I_{\emptyset}=R_{\emptyset}=\emptyset, S_{\emptyset}=\left\{s_{0}\right\}$ and $L_{\emptyset}\left(s_{0}\right)=\emptyset$.

We say that two processes, $P$ and $Q$ are isomorphic if $A C T_{P}=A C T_{Q}, A P_{P}=A P_{Q}$, $I_{P}=I_{Q}$ and there is an isomorphism function $h: S_{P} \rightarrow S_{Q}$ such that $h\left(s_{0 P}\right)=\left(s_{0} Q\right)$, $\left(s_{P}, a_{P}, s_{P}^{\prime}\right) \in R_{P} \Longleftrightarrow\left(h\left(s_{P}\right), a_{P}, h\left(s_{P}^{\prime}\right)\right) \in R_{Q}$ and for every state $s_{P}, L_{P}\left(s_{P}\right)=$ $L_{Q}\left(h\left(s_{P}\right)\right)$.

Lemma 1 For every process $P, P \| \emptyset$ with $N_{P}=\mathcal{I}$ is isomorphic to $P$. 


\subsection{Associativity of Combination}

Combination of processes is commutative. i.e., $P \| Q$ is isomorphic to $Q \| P$. However, combination is not associative in the usual way. When three processes $P, Q$ and $R$ are combined to a network $(P \| Q) \| R$, four renaming functions are needed. First $N_{P}$ and $N_{Q}$ are used to construct $P \| Q$. Then $N_{P \| Q}$ and $N_{R}$ are used to combine $(P \| Q) \| R$. Similarly, to combine $P \|(Q \| R)$, the renaming functions $N_{Q}^{\prime}, N_{R}^{\prime}, N_{Q \| R}^{\prime}$ and $N_{P}^{\prime}$ are needed. The following theorem implies that combinations of a network may be defined such that associativity will hold up to isomorphism.

Theorem 1 (Associativity of Combination) Given a network $(P \| Q) \| R$ combined using $N_{P}, N_{Q}, N_{P \| Q}$, and $N_{R}$, there exist $N_{P}^{\prime}, N_{Q}^{\prime}$ and $N_{R}^{\prime}$ such that the following networks are isomorphic:

1. The given network $(P \| Q) \| R$.

2. $(P \| Q) \| R$ combined using $N_{P}^{\prime}, N_{Q}^{\prime}, N_{R}^{\prime}$, and $N_{P \| Q}^{\prime}=\mathcal{I}$.

3. $P \|(Q \| R)$ combined using $N_{P}^{\prime}, N_{Q}^{\prime}, N_{R}^{\prime}$, and $N_{Q \| R}^{\prime}=\mathcal{I}$.

Proof Let the new renaming functions be defined as follows: $N_{P}^{\prime}=N_{P}, N_{Q}^{\prime}=N_{Q}$, and $N_{R}^{\prime}(a)=N_{P \| Q}^{-1}\left(N_{R}(a)\right)$ for every action $a \in A C T_{R}$.

First let us show that exactly the same ports are paired together by the new renaming functions as were paired by the given ones. Since $N_{P \| Q}^{\prime}=N_{Q \| R}^{\prime}=\mathcal{I}$, we need only to consider the following:

- $N_{P}\left(a_{P}\right)=N_{Q}\left(a_{Q}\right) \Longleftrightarrow N_{P}^{\prime}\left(a_{P}\right)=N_{Q}^{\prime}\left(a_{Q}\right)$

- $N_{P \| Q}\left(N_{Q}\left(a_{Q}\right)\right)=N_{R}\left(a_{R}\right) \Longleftrightarrow N_{Q}\left(a_{Q}\right)=N_{P \| Q}^{-1}\left(N_{R}\left(a_{R}\right)\right) \Longleftrightarrow N_{Q}^{\prime}\left(a_{Q}\right)=N_{R}^{\prime}\left(a_{R}\right)$

- $N_{P \| Q}\left(N_{P}\left(a_{P}\right)\right)=N_{R}\left(a_{R}\right) \Longleftrightarrow N_{P}\left(a_{P}\right)=N_{P \| Q}^{-1}\left(N_{R}\left(a_{R}\right)\right) \Longleftrightarrow N_{P}^{\prime}\left(a_{P}\right)=N_{R}^{\prime}\left(a_{R}\right)$

This directly implies the isomorphism of the first and second networks. Moreover, the above implies that $N_{P}^{\prime}, N_{Q}^{\prime}$ and $N_{R}^{\prime}$ are sufficient to determine the pairing of ports regardless of the order in which the processes $P, Q$ and $R$ are combined.

Now we define a mapping function from states of $(P \| Q) \| R$ to states of $P \|(Q \| R)$ and show that the two networks are isomorphic with respect to that function. Let the isomorphism function $h: S_{(P \| Q) \| R} \rightarrow S_{P \|(Q \| R)}$ be:

$$
h\left(\left(\left(s_{P}, s_{Q}\right), s_{R}\right)\right)=\left(s_{P},\left(s_{Q}, s_{R}\right)\right)
$$

To show that $P \|(Q \| R)$ and $(P \| Q) \| R$ are isomorphic, we consider only the requirements on the transition relations, as all others are immediate. Since there are no unpaired ports in either network, all the transitions are labeled by $\epsilon$, and $R_{(P \| Q) \| R}$ contains six kinds of transitions: Internal transitions of either $P, Q$ or $R$, and communication transitions involving either $P$ and $Q, P$ and $R$, or $Q$ and $R$. For similar reasons, $R_{P \|(Q \| R)}$ contains the same six kinds of transitions. By looking at each of the possible kinds of transitions, we can see that 


$$
\begin{aligned}
& \left(\left(\left(s_{P}, s_{Q}\right), s_{R}\right), \epsilon,\left(\left(s_{P}^{\prime}, s_{Q}^{\prime}\right), s_{R}^{\prime}\right)\right) \in R_{(P \| Q) \| R} \Longleftrightarrow \\
& \left(\left(s_{P},\left(s_{Q}, s_{R}\right)\right), \epsilon,\left(s_{P}^{\prime},\left(s_{Q}^{\prime}, s_{R}^{\prime}\right)\right)\right) \in R_{P \|(Q \| R)} \Longleftrightarrow \\
& \left(h\left(\left(\left(s_{P}, s_{Q}\right), s_{R}\right)\right), \epsilon, h\left(\left(\left(s_{P}^{\prime}, s_{Q}^{\prime}\right), s_{R}^{\prime}\right)\right)\right) \in R_{P \|(Q \| R)}
\end{aligned}
$$

\subsection{Network Grammars}

The formalism we suggest for the description of distributed finite-state algorithms is a Network Grammar, $G=(\Sigma, \Delta, \mathcal{P}, T) . \Sigma^{\star}$ is the set of networks combined of instances of process in $\Sigma$. The grammar defines a subset of networks combined using the production rules.

- The terminals, $\Sigma$, are process types, which are processes with a singleton set of indices.

- The nonterminals, $\Delta$, are subnetwork types, each of which represents an unspecified process with a specified set of action names. For a subnetwork type $A$, this set is denoted by $A C T_{A}$.

- Subnetwork types $B$ and $C$ may be combined together using renaming functions $N_{B}$ and $N_{C}$, defined over $A C T_{B}$ and $A C T_{C}$, respectively. Similar to process combination, the renaming functions specify those action names (ports) in $B$ and $C$ that are paired together. $A C T_{B \| C}$, the set of actions names of $B \| C$, contains all the unpaired action names of $B$ and $C$ after renaming.

- $\mathcal{P}$ is the set of production rules. A production rule in a network grammar specifies how subnetwork types and process types should be combined. For $A \in \triangle, B, C \in$ $(\Sigma \cup \Delta),\left(A,\left(B, N_{B}\right),\left(C, N_{C}\right)\right)$, abbreviated to $A \rightarrow B \| C$, describes a derivation in which $A$ is replaces by $B \| C$. It is required that $A C T_{A}=A C T_{B \| C}$. For simplicity we assume that each production rule is a binary rule of the form $A \rightarrow B \| C$. A unary production rule may be obtained by replacing $C$ with the empty process. It is not allowed for both $B$ and $C$ to be $\emptyset$.

- The start symbol $T$ represents a subnetwork type with an empty set of action names.

Each instance of a process type has a unique index value. Variables of $G$, other than $T$, can derive processes with unpaired ports.

We consider only grammars in which every symbol is reachable, and derives at least one subnetwork of terminals.

We used our formalism to define algorithms for a family of networks with nontrivial topologies. 


\section{The logic $\mathbf{L T L}^{2}$}

$\mathrm{LTL}^{2}$ is a two-leveled linear time temporal logic. It is defined by means of two types of formulas: local and global. Let $A P$ be a set of atomic propositions, which are indexed by a set of index variables $I V$. A local formula is either:

- $a_{i}$, if $a \in A P$ and $i \in I V$.

- $\neg f, f \vee g$ and $f \mathrm{U} g$, if $f$ and $g$ are local formulas.

A global formula is either:

- $\bigvee_{i} \mathbf{A} f(i)$ and $\bigvee_{i} \mathbf{E} f(i)$, if $f(i)$ is a local formula that has exactly one free index variable $i$. We will refer to this type of global formulas as basic formulas. Basic formulas have no free index variables.

- $\neg f, f \vee g$ and $f \mathrm{U} g$, if $f$ and $g$ are global formulas.

An $\mathrm{LTL}^{2}$ formula is either $\mathbf{A} f$ or $\mathbf{E} f$, where $f$ is a global formula.

We define the semantics of $\mathrm{LTL}^{2}$ with respect to a Kripke structure

$$
K=\left\langle A P, I, S, R, s_{0}, L\right\rangle
$$

where

- $A P$ is the set of atomic propositions.

- $I$ is the set of index values.

- $S$ is the set of states.

- $R \subseteq S \times S$ is the transition relation, which must be total in both of its arguments. We write $s_{1} \rightarrow s_{2}$ to indicate that $\left(s_{1}, s_{2}\right) \in R$.

- $s_{0}$ is the initial state.

- $L: S \rightarrow 2^{(A P \times I)}$ is the indexed proposition labeling.

We define a path in $K$ to be a sequence of states, $\pi=s_{1}, s_{2}, \ldots$ such that for every $i \geq 1, s_{i} \rightarrow s_{i+1}$. $\pi^{i}$ will denote the suffix of $\pi$ starting in $s_{i}$, and first $(\pi)$ will denote the first state in $\pi$. We only consider infinite paths in $K$.

We distinguish between two types of formulas. Formulas of the form $\neg f, f \vee g$ or $f \mathbf{U}_{g}$ are path formulas. Formulas of the form $a_{i}, \mathbf{A} f, \mathbf{E} f$ or $\bigvee_{i} f$ are state formulas. We use the notation $K, \pi, J \models f(K, s, J \models f)$ to denote that the formula $f$ holds along path $\pi$ (in a state $s$ ) for a subset $J \neq \emptyset$ of the index values in the structure $K$. The relation $\models$ is defined inductively as follows: 


$$
\begin{aligned}
& K, s,\{i\} \vDash a_{i} \quad \Leftrightarrow a_{i} \in L(s) . \\
& K, \pi,\{i\} \models a_{i} \quad \Leftrightarrow \quad K, \operatorname{first}(\pi),\{i\} \models a_{i} \\
& K, \pi, J \models \neg f \quad \Leftrightarrow \quad K, \pi, J \not \models f . \\
& K, \pi, J \vDash f \vee g \Leftrightarrow K, \pi, J \models f \text { or } K, \pi, J \models g . \\
& K, \pi, J \models f \mathrm{U} g \Leftrightarrow \text { there exists a } l \geq 1 \text { such that } K, \pi^{l}, J \models g \\
& \text { and for all } 1 \leq j<l \quad K, \pi^{j}, J \models f \text {. } \\
& K, \pi, J \models \bigvee_{i} f \Leftrightarrow K, f i r s t(\pi), J \models \bigvee_{i} f \text {. } \\
& K, s, J \models \bigvee_{i} f \Leftrightarrow \text { there exists an } i_{0} \in J \text { such that } K, s,\left\{i_{0}\right\} \models f \text {. } \\
& K, s, J \models \mathbf{A} f \Leftrightarrow \text { for every path } \pi \text { starting in } s \quad K, \pi, J \models f \text {. } \\
& K, s, J \models \mathbf{E} f \Leftrightarrow \text { there exists a path } \pi \text { starting in } s \text { such that } K, \pi, J \models f .
\end{aligned}
$$

We will use the following abbreviations:

$$
\bigwedge_{i} \mathbf{A} f=\neg \bigvee_{i} \mathbf{E} \neg f, \quad \bigwedge_{i} \mathbf{E} f=\neg \bigvee_{i} \mathbf{A} \neg f, \quad \mathbf{F} f=\text { true } \mathbf{U} f \text { and } \quad \mathbf{G} f=\neg \mathbf{F} \neg f
$$

For a formula $f \in \mathrm{LTL}^{2}$, we define $B F(f)$ as the set of basic formulas in $f$. Let $\mathcal{F}$ be some set of basic formulas over a set $A P_{\mathcal{F}}$ of atomic propositions. Given two structures $K, K^{\prime}$, such that $A P_{K}=A P_{K^{\prime}} \supseteq A P_{\mathcal{F}}$ and two index sets $J_{K}$ and $J_{K^{\prime}}$, such that $J_{K} \subseteq I_{K}$ and $J_{K^{\prime}} \subseteq I_{K^{\prime}}$, we define two notions of equivalence with respect to $\mathcal{F}$.

Specification equivalence: $\left(K, J_{K}\right) \equiv_{\mathcal{F}}\left(K^{\prime}, J_{K^{\prime}}\right)$ if and only if

$$
\forall f[B F(f) \subseteq \mathcal{F}]: K, s_{0 K}, J_{K} \vDash f \Longleftrightarrow K^{\prime}, s_{0} K^{\prime}, J_{K^{\prime}} \models f
$$

Computation equivalence: $\left(K, J_{K}\right) \mathbf{C}_{\mathcal{F}}\left(K^{\prime}, J_{K^{\prime}}\right)$ if and only if

1. For every path $\pi$ starting in $s_{0 K}$ there is a path $\pi^{\prime}$ starting in $s_{0 K^{\prime}}$, partitions of both paths $B_{1}, B_{2}, \ldots, B_{1}^{\prime}, B_{2}^{\prime}, \ldots$ such that for every $j, B_{j}$ and $B_{j}^{\prime}$ are both nonempty and finite, and for every state $s$ in $B_{j}$, every state $s^{\prime}$ in $B_{j}^{\prime}$, and for every $f \in \mathcal{F}$,

$$
K, s, J_{K} \models f \Longleftrightarrow K^{\prime}, s^{\prime}, J_{K^{\prime}} \models f
$$

2. For every path $\pi^{\prime}$ starting in $s_{0} K^{\prime}$ there is a path $\pi$ starting in $s_{0 K}$ that satisfies the same conditions as above.

Theorem $2\left(K, J_{K}\right) \equiv_{\mathcal{F}}\left(K^{\prime}, J_{K^{\prime}}\right)$ if and only if $\left(K, J_{K}\right) \mathbf{C}_{\mathcal{F}}\left(K^{\prime}, J_{K^{\prime}}\right)$.

Proof First we note that once we refer to the basic formulas as atomic propositions, LTL $^{2}$ is reduced to a variant of LTL without the next-time operator. This variant also includes a single top level path quantifier which is either $\mathbf{A}$ ("for all paths") or $\mathbf{E}$ ("there exists a path").

We show that both the specification equivalence and the computation equivalence correspond to LTL-equivalence of structures labeled by the basic formulas in $\mathcal{F}$. 
We define a function $h_{\mathcal{F}}$ that transforms a pair $\left(K, J_{K}\right)$ to a structure (without an index set) labeled by $\mathcal{F}$.

$$
h_{\mathcal{F}}\left(K, J_{K}\right)=\left\langle\mathcal{F}, S, R, s_{0}, L_{\mathcal{F}}\right\rangle
$$

where $L_{\mathcal{F}}(s)=\left\{f \mid f \in \mathcal{F}\right.$ and $\left.K, s, J_{K} \models f\right\}$.

According to the semantics of LTL ${ }^{2}$ and LTL:

$$
\begin{gathered}
\forall f[B F(f) \subseteq \mathcal{F}]: K, s, J_{K} \models_{\mathrm{LTL}^{2}} f \Longleftrightarrow h_{\mathcal{F}}\left(K, J_{K}\right), s \models_{\mathrm{LTL}} f \\
\left(K, J_{K}\right) \equiv_{\mathcal{F}}\left(K^{\prime}, J_{K^{\prime}}\right) \text { if and only if } h_{\mathcal{F}}\left(K, J_{K}\right) \equiv_{\mathrm{LTL}} h_{\mathcal{F}}\left(K^{\prime}, J_{K^{\prime}}^{\prime}\right)
\end{gathered}
$$

On the other hand, using a stuttering equivalence relation for LTL derived from the equivalence for PTL suggested in [SG], $h_{\mathcal{F}}\left(K, J_{K}\right) \equiv_{\mathrm{LTL}} h_{\mathcal{F}}\left(K^{\prime}, J_{K}^{\prime}\right)$ if and only if

1. For every path $\pi$ starting in $s_{0 K}$ there is a path $\pi^{\prime}$ starting in $s_{0} K^{\prime}$, partitions of both paths $B_{1}, B_{2}, \ldots, B_{1}^{\prime}, B_{2}^{\prime}, \ldots$ such that for every $j, B_{j}$ and $B_{j}^{\prime}$ are both nonempty and finite, and for every state $s$ in $B_{j}$, every state $s^{\prime}$ in $B_{j}^{\prime}, L_{\mathcal{F}}(s)=L_{\mathcal{F}}^{\prime}\left(s^{\prime}\right)$.

2. For every path $\pi^{\prime}$ starting in $s_{0} K^{\prime}$ there is a path $\pi$ starting in $s_{0} K$ that satisfies the same conditions as above.

Therefore $\left(K, J_{K}\right) \equiv_{\mathcal{F}}\left(K^{\prime}, J_{K^{\prime}}\right)$ if and only if $\left(K, J_{K}\right) \mathbf{C}_{\mathcal{F}}\left(K^{\prime}, J_{K^{\prime}}\right)$.

It will sometime be convenient to refer to a network in a context which requires a structure instead. When this happens, the required structure is the one obtained from the network by omitting the transition labels (all of which are $\epsilon$ ). Since Kripke structures have total transition relations, so must the networks.

In the remainder of the paper we refer only to finite Kripke structures.

\section{The Communication Behavior}

The communication behavior of a process $P$ given an environment $Q$, is denoted by $P: Q$. Similarly to $P \| Q$, it is defined by means of $P, Q, N_{P}$ and $N_{Q}$, but only in case the combination of $P \| Q$, using $N_{P}$ and $N_{Q}$, results in a network (with no unpaired ports). The main differences between $P \| Q$ and $P: Q$ are:

- Transitions of $P: Q$ are labeled by $\epsilon, \bar{\epsilon}$ or actions in $A C T_{P}$.

- A state $s$ in $P: Q$ is labeled by basic formulas, rather than atomic propositions.

- $P: Q$ has two special states sink and $\overline{\operatorname{sink}}$. All communications on channels between $P$ and $Q$ in which $P$ is willing to participate while $Q$ is not, are directed to sink. Similarly, communications in which $Q$ is willing to participate but $P$ is not, are directed to $\overline{\operatorname{sink}}$. This records the mutual influence of the environment $Q$ and the process $P$ by means of communication enableness.

Given $P, Q, N_{P}$ and $N_{Q}$ such that $N_{P}\left(A C T_{P}\right)=N_{Q}\left(A C T_{Q}\right)$, the communication behavior $P: Q$ is formally defined as follows:

$$
P: Q=\left\langle A C T_{P: Q}, B F_{P: Q}, S_{P: Q}, R_{P: Q}, s_{O P: Q}, M_{P: Q}\right\rangle
$$

where 
- $A C T_{P: Q}=A C T_{P}$

- $B F_{P: Q}$ is a set of basic formulas, defined over $A P_{P}$, the set of atomic propositions of $P$.

- $S_{P: Q}=\{\operatorname{sink}, \overline{\operatorname{sink}}\} \cup\left(S_{P} \times S_{Q}\right)$ where sink and $\overline{\operatorname{sink}}$ are special states.

- $M_{P: Q}\left(\left(s_{P}, s_{Q}\right)\right)=\left\{f \mid f \in B F_{P: Q}\right.$ and $\left.P \| Q,\left(s_{P}, s_{Q}\right), I_{P} \models f\right\}$

- $M_{P: Q}(\operatorname{sink})=i \operatorname{ssink}$

- $M_{P: Q}(\overline{\operatorname{sink}})=\overline{i s \operatorname{sink}}$

- $s_{0 P: Q}=\left(s_{0 P}, s_{0} Q\right)$

In order to define $R_{P: Q}$ we need the following definitions:

- A path containing only $\epsilon$ transitions of $Q$ is called an $\epsilon$-path in $Q$.

- $\epsilon$-closure $(s)$ is the set of states that are reachable from $s$ by an $\epsilon$-path.

$$
\begin{array}{ll}
R_{P: Q}= & \\
\left\{\left(\left(s_{P}, s_{Q}\right), a_{P},\left(s_{P}^{\prime}, s_{Q}^{\prime}\right)\right)\right. & \mid\left(s_{P}, a_{P}, s_{P}^{\prime}\right) \in R_{P} \text { and }\left(s_{Q}, a_{Q}, s_{Q}^{\prime}\right) \in R_{Q}, \\
& \text { where } \left.N_{P}\left(a_{P}\right)=N_{Q}\left(a_{Q}\right)\right\} \cup \\
\left\{\left(\left(s_{P}, s_{Q}\right), \epsilon,\left(s_{P}^{\prime}, s_{Q}\right)\right)\right. & \left.\mid\left(s_{P}, \epsilon, s_{P}^{\prime}\right) \in R_{P}\right\} \cup \\
\left\{\left(\left(s_{P}, s_{Q}\right), \bar{\epsilon},\left(s_{P}, s_{Q}^{\prime}\right)\right)\right. & \left.\mid\left(s_{Q}, \epsilon, s_{Q}^{\prime}\right) \in R_{Q}\right\} \cup \\
\left\{\left(\left(s_{P}, s_{Q}\right), a_{P}, \operatorname{sink}\right)\right. & \mid \exists\left(s_{P}, a_{P}, s_{P}^{\prime}\right) \in R_{P}: a_{P} \neq \epsilon \text { and } \\
& \forall s_{Q}^{\prime} \in \epsilon-\operatorname{closure}\left(s_{Q}\right) \forall\left(s_{Q}^{\prime}, a_{Q}, s_{Q}^{\prime \prime}\right) \in R_{Q}: \\
& \left.N_{P}\left(a_{P}\right) \neq N_{Q}\left(a_{Q}\right)\right\} \cup \\
\left\{\left(\left(s_{P}, s_{Q}\right), a_{P}, \overline{\operatorname{sink}}\right)\right. & \mid \exists\left(s_{Q}, a_{Q}, s_{Q}^{\prime}\right) \in R_{Q}: N_{P}\left(a_{P}\right)=N_{Q}\left(a_{Q}\right) \text { and } \\
& \left.\forall\left(s_{P}^{\prime}, a_{P}, s_{P}^{\prime \prime}\right) \in R_{P}: s_{P}^{\prime} \notin \epsilon \text {-closure }\left(s_{P}\right)\right\} \cup \\
\{(\operatorname{sink}, \epsilon, \operatorname{sink})\} \cup & \\
\{(\overline{\operatorname{sink}}, \bar{\epsilon}, \overline{\operatorname{sink}})\} &
\end{array}
$$

Explanation: $R_{P: Q}$ is a union of 7 sets of transitions. The first one records the transitions in which $P$ and $Q$ cooperate. The second and the third contain internal transitions of $P$ and $Q$, respectively. The fourth records the communications which are enabled by $P$ but disabled by $Q$ in the current state, and all the states in its $\epsilon$-closure. The fifth set records the dual case, in which $Q$ enables the communication while $P$ disables it. The transition in both cases is labeled by an action in $A C T_{P}$. The sixth and seventh sets contain self loops for sink and $\overline{\operatorname{sink}}$. 
Lemma $2\left(\left(s_{P}, s_{Q}\right), \alpha,\left(s_{P}^{\prime}, s_{Q}^{\prime}\right)\right) \in R_{P: Q}$ if and only if $\left(\left(s_{P}, s_{Q}\right), \epsilon,\left(s_{P}^{\prime}, s_{Q}^{\prime}\right)\right) \in R_{P \| Q}$. i.e., every transition in $P: Q$ that does not result in either $\operatorname{sink}$ or $\overline{\operatorname{sink}}$ has a corresponding transition in $P \| Q$, and vice versa.

\section{Proof}

- By the definition of $R_{P: Q}$, a transition, $\left(\left(s_{P}, s_{Q}\right), \alpha,\left(s_{P}^{\prime}, s_{Q}^{\prime}\right)\right) \in R_{P: Q}$ if and only if one of the following conditions holds:

1. $\left(s_{P}, a_{P}, s_{P}^{\prime}\right) \in R_{P}$ and $\left(s_{Q}, a_{Q}, s_{Q}^{\prime}\right) \in R_{Q}$, where $N_{P}\left(a_{P}\right)=N_{Q}\left(a_{Q}\right)$

2. $\left(s_{P}, \epsilon, s_{P}^{\prime}\right) \in R_{P}$ and $s_{Q}=s_{Q}^{\prime}$

3. $\left(s_{Q}, \epsilon, s_{Q}^{\prime}\right) \in R_{Q}$ and $s_{P}=s_{P}^{\prime}$

- Because $P \| Q$ is a network, and by the definition of $R_{P \| Q},\left(\left(s_{P}, s_{Q}\right), \epsilon,\left(s_{P}^{\prime}, s_{Q}^{\prime}\right)\right) \in$ $R_{P \| Q}$ if and only if one of the above conditions holds.

- Therefore, $\left(\left(s_{P}, s_{Q}\right), \alpha,\left(s_{P}^{\prime}, s_{Q}^{\prime}\right)\right) \in R_{P: Q}$ if and only if $\left(\left(s_{P}, s_{Q}\right), \epsilon,\left(s_{P}^{\prime}, s_{Q}^{\prime}\right)\right) \in R_{P \| Q}$.

We define $\pi=s_{0} \stackrel{\alpha_{3}}{\rightarrow} s_{1} \stackrel{\alpha_{2}}{\rightarrow} s_{2} \rightarrow \cdots$ to be a path in $P: Q$ if $\forall i \geq 0\left(s_{i}, \alpha_{i+1}, s_{i+1}\right) \in R_{P: Q}$ Corollary 1 For every path in $P: Q$ from the initial state, $s_{0} \stackrel{\alpha_{1}}{\rightarrow} s_{1} \stackrel{\alpha_{2}}{\rightarrow} s_{2} \rightarrow \cdots$, with $\alpha_{i} \in A C T_{P} \cup\{\epsilon, \bar{\epsilon}\}$, that does not contain sink or $\overline{\operatorname{sink}}, s_{0}, s_{1}, s_{2}, \cdots$ is a path in $P \| Q$.

Corollary 2 For every path in $P \| Q$ from the initial state, $s_{0}, s_{1}, s_{2}, \cdots$ there exists a sequence of actions $\alpha_{1}, \alpha_{2}, \alpha_{3}, \ldots$ where $\alpha_{i} \in A C T_{P} \cup\{\epsilon, \bar{\epsilon}\}$ such that $s_{0} \stackrel{\alpha_{1}}{\rightarrow} s_{1} \stackrel{\alpha_{2}}{\rightarrow} s_{2} \rightarrow \cdots$ is a path in $P: Q$.

\subsection{Equivalence of Communication Behaviors}

Two communication behaviors $P: Q$ and $P^{\prime}: Q^{\prime}$ are equivalent $\left(P: Q \equiv P^{\prime}: Q^{\prime}\right)$ if

1. $A C T_{P: Q}=A C T_{P^{\prime}: Q^{\prime}}$

2. $B F_{P: Q}=B F_{P^{\prime}: Q^{\prime}}$

3. For every path $\pi$ starting in $s_{0} P: Q$ there is a path $\pi^{\prime}$ starting in $s_{0} P^{\prime}: Q^{\prime}$ and partition of both paths $B_{1}, B_{2}, \ldots, B_{1}^{\prime}, B_{2}^{\prime}, \ldots$ such that for every $j$ :

(a) $B_{j}$ and $B_{j}^{\prime}$ are both finite, non-empty and contain only $\epsilon$ and $\bar{\epsilon}$ transitions.

(b) For every state $s$ in $B_{j}$ and every state $s^{\prime}$ in $B_{j}^{\prime} \quad M_{P: Q}(s)=M_{P^{\prime}: Q^{\prime}}\left(s^{\prime}\right)$.

(c) If last $\left(B_{j}\right) \stackrel{\alpha_{j}}{\rightarrow} \operatorname{first}\left(B_{j+1}\right)$ then last $\left(B_{j}^{\prime}\right) \stackrel{\alpha_{j}}{\rightarrow} \operatorname{first}\left(B_{j+1}^{\prime}\right)$.

4. For every path $\pi^{\prime}$ starting in $s_{0} P^{\prime}: Q^{\prime}$ there is a path $\pi$ starting in $s_{0 P: Q}$ that satisfies the same conditions as in 3 .

Lemma 3 If $P: Q \equiv P^{\prime}: Q^{\prime}$ then $\left(P \| Q, I_{P}\right) \equiv_{B F_{P}: Q}\left(P^{\prime} \| Q^{\prime}, I_{P^{\prime}}\right)$ 
Proof First we show that if $P: Q \equiv P^{\prime}: Q^{\prime}$ then $\left(P \| Q, I_{P}\right) \mathbf{C}_{B F_{P: Q}}\left(P^{\prime} \| Q^{\prime}, I_{P^{\prime}}\right)$.

- $P: Q \equiv P^{\prime}: Q^{\prime}$ therefore $B F_{P: Q}=B F_{P^{\prime}: Q^{\prime}}$.

- The initial states of $P: Q$ and $P \| Q$ are both $\left(s_{0} P, s_{0} Q\right)$, and thus are equal. Similarly, the initial states of $P^{\prime}: Q^{\prime}$ and $P^{\prime} \| Q^{\prime}$ are equal, too.

- Let $\pi=s_{0}, s_{1}, s_{2}, \cdots$ be a path in $P \| Q$ starting in the initial state $s_{0} P \| Q$.

By corollary 1 there is a path $s_{0} P: Q \stackrel{\alpha_{l}}{\rightarrow} s_{1} \stackrel{\alpha_{2}}{\rightarrow} s_{2} \rightarrow \cdots$ in $P: Q$.

By the equivalence of $P: Q$ and $P^{\prime}: Q^{\prime}$ there is a path $s_{0} P^{\prime}: Q^{\prime} \stackrel{\beta_{1}}{\rightarrow} s_{1}^{\prime} \stackrel{\beta_{2}}{\rightarrow} s_{2}^{\prime} \rightarrow \cdots$ in $P^{\prime}: Q^{\prime}$, and a partitioning of both paths that satisfy conditions 3 of the equivalence definition above.

By corollary $2, \pi^{\prime}=s_{0} P^{\prime} \| Q^{\prime}, s_{1}^{\prime}, s_{2}^{\prime}, \cdots$ is a path in $P^{\prime} \| Q^{\prime}$. The partition $B_{1}, B_{2}, \ldots$ and $B_{1}^{\prime}, B_{2}^{\prime}, \ldots$, applied to $\pi$ and $\pi^{\prime}$ respectively, satisfy the conditions of computation equivalence of Kripke structures with respect to $B F_{P: Q}$.

- Similarly, for every a path in $P^{\prime} \| Q^{\prime}$, starting in the initial state, there exists a path in $P \| Q$ that satisfies the conditions of computation equivalence of Kripke structures with respect to $B F_{P: Q}$.

Thus, $\left(P \| Q, I_{P}\right) \mathbf{C}_{B F_{P: Q}}\left(P^{\prime} \| Q^{\prime}, I_{P^{\prime}}\right)$, and by Theorem $2,\left(P \| Q, I_{P}\right) \equiv_{B F_{P}: Q}\left(P^{\prime} \| Q^{\prime}, I_{P^{\prime}}\right)$.

Theorem 3 (3-Way Orthogonality) Let all the communication behaviors be defined over a fixed set of basic formulas. If $P^{\prime}:(Q \| R) \equiv P:(Q \| R), Q^{\prime}:(P \| R) \equiv Q:(P \| R)$ and $R^{\prime}:(P \| Q) \equiv R:(P \| Q)$ then $P^{\prime}:\left(Q^{\prime} \| R^{\prime}\right) \equiv P:(Q \| R)$.

Essentially, the theorem says that it is sufficient to check the replacement of each component of a communication behavior separately to conclude that replacing all components at the same time results in an equivalent communication behavior. Due to lack of space, the proof is omitted.

Corollary 3 If $P^{\prime}: Q \equiv P: Q$ and $Q^{\prime}: P \equiv Q: P$ then $P^{\prime}: Q^{\prime} \equiv P: Q$.

Corollary 4 If $P^{\prime}: Q \equiv P: Q$ then $Q: P^{\prime} \equiv Q: P$.

\subsection{Verification of Algorithms}

Verification of an algorithm means to verify all the programs it describes. The purpose of this section is to establish a theoretical basis for automatic verification of algorithms defined by network grammars. Let $G=(\Sigma, \Delta, \mathcal{P}, T)$ be a description of an algorithm, and let SPEC $\in \mathrm{LTL}^{2}$ be a specification to be verified for that algorithm. With every terminal and nonterminal symbol $W \in \Sigma \cup \Delta$ we associate two representative processes $w, \bar{w} \in \Sigma^{\star}$. $w$ is the representative of the subnetworks derived from $W$ by the grammar, while $\bar{w}$ is the representative of the possible environments for these subnetworks. For terminal symbols $W \in \Sigma, w$ is (an instance of) process type $W$. The environment representative, $\bar{t}$, of the start symbol $T$ is the empty process $\emptyset$.

From now on, we only consider communication behaviors labeled by $B F(\mathrm{SPEC})$. 
Lemma 4 If for every production rule $A \rightarrow B \| C$,

$$
\forall b^{\prime}, c^{\prime} \in \Sigma^{\star}\left[\text { if } b^{\prime}: \bar{b} \equiv b: \bar{b} \wedge c^{\prime}: \bar{c} \equiv c: \bar{c} \text { then } a: \bar{a} \equiv\left(b^{\prime} \| c^{\prime}\right): \bar{a}\right]
$$

then for every $W \in \Sigma \cup \Delta$, and every $w^{\prime} \in \Sigma^{\star}$ such that $W \Rightarrow^{\star} w^{\prime}, w^{\prime}: \bar{w} \equiv w \cdot \bar{w}$.

The proof is by induction on the number of productions in the derivation of $\boldsymbol{w}^{\prime}$.

Lemma 5 For every production rule $A \rightarrow B \| C$, if $a: \bar{a} \equiv(b \| c): \bar{a}, \bar{b}: \bar{b} \equiv(\bar{a} \| c): b$ and $\bar{c}: c \equiv(\bar{a} \| b): c$, then $\forall b^{\prime}, c^{\prime} \in \Sigma^{\star}\left[\right.$ if $b^{\prime}: \bar{b} \equiv b: \bar{b} \wedge c^{\prime}: \bar{c} \equiv c: \bar{c}$ then $\left.a: \bar{a} \equiv\left(b^{\prime} \| c^{\prime}\right): \bar{a}\right]$

The proof is based on theorem 3 .

Theorem 4 (Consistent Grammars) If for every production rule $A \rightarrow B \| C, a: \bar{a} \equiv$ $(b \| c): \bar{a}, \bar{b}: b \equiv(\bar{a} \| c): b$ and $\bar{c}: c \equiv(\bar{a} \| b): c$, then

$$
\left(t, s_{0} t, I_{t}=S P E C\right) \Leftrightarrow \forall t^{\prime} \in \Sigma^{\star}\left[T \Rightarrow^{\star} t^{\prime}\right]\left(t^{\prime}, s_{0} t^{\prime}, I_{t^{\prime}} \models S P E C\right)
$$

The proof is based on lemma 4, lemma 5 and the definition of specification equivalence.

A network grammar is consistent if is satisfies the conditions of theorem 4. Once the consistency of a grammar is established, the representative $t$, of the start symbol $T$, satisfies SPEC if and only if the algorithm does.

\section{The Verification Procedure}

Given a description of an algorithm by means of a network grammer $G=(\Sigma, \Delta, \mathcal{P}, T)$, and a specification SPEC, the verification procedure consists of the following steps:

1. Find $B F(\mathrm{SPEC})$, the set of basic formulas in SPEC.

2. For every $W \in \Sigma \cup \Delta$ choose a representative $w \in \Sigma^{\star}$ :

(a) For every terminal, its representative is the process type associated with it.

(b) For any symbol $A$ that does not have a representative, if there is a production rule $A \rightarrow B \| C$, for which both $B$ and $C$ have representatives, then $a=b \| c$ is the representative of $A$.

(c) Repeat step $2 \mathrm{~b}$ until every symbol has a representative.

3. For $W \in \Sigma \cup \Delta$ choose an environment representative $\bar{w} \in \Sigma^{\star}$ :

(a) For the start symbol $T$, the representative is the empty process $\emptyset$.

(b) For any symbol $B$ that does not have an environment representative, if there is a production rule $A \rightarrow B \| C$ (or $A \rightarrow C \| B$ ), for which $A$ has an environment representative $\bar{a}$, then $\bar{b}=\bar{a} \| c$ is the environment representative of $B$.

(c) Repeat step $3 \mathrm{~b}$ until every symbol has an environment representative. 
4. For every production rule $A \rightarrow B \| C$ construct the communication behavior of $a: \bar{a}, \bar{b}: b, \bar{c}: c,(b \| c): \bar{a},(\bar{a} \| c): b,(\bar{a} \| b): c$ labeled by basic formulas in $B F(\mathrm{SPEC})$. The labeling is determined using an LTL model checker.

5. If $G$ is consistent then apply an LTL model checker to $t: \emptyset$. The algorithm satisfies SPEC if and only if $t: \emptyset$ satisfies SPEC.

6. If $G$ is not consistent, out method is incompetent with the verification of the grammar.

\section{Acknowledgements}

We thank Nissim Francez and Amir Pnueli for helpful discussions and hints.

The second author, O. Grumberg, was partly supported by the V.P.R. fund - Loewengart Research Fund.

\section{References}

[B] M.C. Browne, "An Improved Algorithm for the Automatic Verification of Finite State Systems using Temporal Logic", Proc. of the 1986 Conference on Logic in Computer Science, Cambridge, MA, pp. 260-267, June 1986.

[BCDM] M.C. Browne, E.M. Clarke, D. Dill, and B. Mishra, "Automatic Verification of Sequential Circuits using Temporal Logic", IEEE Trans. on Computers, C-35(12), December 1986.

[CBBG] E.M. Clarke, S. Bose, M.C. Browne, and O. Grumberg, "The design and verification of finite-state hardware controllers", conference on VLSI, Taiwan, 1987.

[CG] E.M. Clarke, and O. Grumberg, "Avoiding the State Explosion in Temporal Logic Model Checking Algorithms", Proc. of the 6th Annual Symposium on Principles of Distributed Computing, ACM, pp. 294-303, August 1987.

[CGB] E.M. Clarke, O. Grumberg, and M.C. Browne, "Reasoning about Networks with Many Identical Finite-State Processes", Information and Computations, 81(1), pp. 13-31, 1989.

[CES] E.M. Clarke, E.A. Emerson, and A.P. Sistla, "Automatic Verification of FiniteState Concurrent Systems using Temporal Logic Specifications", ACM Trans. on Programming Languages and Systems, 8(2), pp. 244-263, 1986.

[D] E. Dijkstra, Invariance and Non-Determinacy, (C.A.R. Hoare, and J.C. Shepherson, eds.), Mathematical Logic and Programming Languages, pp. 157-163, Prentice-Hall, 1985.

[DC] D. Dill, and E.M. Clarke, "Automatic Verification of Asynchronous Circuits using Temporal Logic", IEEE Proc., 133, pt. E(5), September 1986. 
[EL] E.A. Emerson, and C.L. Lei, "Modalities for Model Checking: Branching Time Strikes Back," Conf. Record of the 20th Annual ACM Symp. on Principles of Programming Languages, New Orleans, La., January 1985.

[F] N. Francez, "Distributed Termination", ACM-TOPLAS 2, 1, pp. 42-55, 1980.

[FRS] N. Francez, M. Rodeh, and M. Sintzoff, "Distributed Termination with Interval Assertions", Proc, of the Inf. Col. on Formalization of Programming Concepts, Peninscula, Spain, LNCS 107, Springer-Verlag 1981.

[I] INMOS Limited, OCCAM Programming Manual, Prentice-Hall, 1984.

[L] G. LeLann, "Distributed Systems - Towards a Formal Approach", Information Processing, 77, pp. 155-160, North-Holland, Amsterdam.

[LP] O. Lichtenstein, and A. Pnueli, "Checking that Finite State Concurrent Programs Satisfy their Linear Specification", Conf. Record of the 20th Annual ACM Symp. on Principles of Programming Languages, New Orleans, La., January 1985 .

[M] R. Milner, A Calculus of Communicating Systems, LNCS 92, Springer-Verlag 1980 .

[MC] B. Mishra, and E.M. Clarke, "Hierarchical Verification of Asynchronous Circuits using Temporal Logic", Theoretical Computer Science, 38, pp. 269-291, 1985.

[QS] J.P. Quielle, and J. Sifakis, "Specification and Verification of Concurrent Systmes in CESAR", Proc. of the 5th Inter. Symp. on Programming, 1981.

[SC] A.P. Sistla, and E.M. Clarke, "Complexity of Propositional Temporal Logics", J. of the Association for Computing Machinery, 32(3), pp. 733-749, July 1986.

[SG] A.P. Sistla, and S. German, "Reasoning with Many Processes", Proc, of the Symp. on Logic in Computer Science, Ithaca, N.Y., June 1987.

[RRSV] J.L. Richier, C. Rodriguez, J. Sifakis, and J. Voiron, "XESAR - User's Guide", Technical Report, IMAG, Institut National Polytechnique de Grenoble, September 1987. 